\title{
West Greenland Peregrine Falcon Survey, 1978
}

WILLIAM G. MATTOX ${ }^{1}$, WILLIAM R. HEINRICH ${ }^{2}$, JACK OAR ${ }^{3}$, STEVEN J. BELARDO ${ }^{4}$, KENTON E. RIDDLE ${ }^{5}$ and THOMAS $M$. SMYLIE $^{6}$

The project began in 1972 when 9 active cliffs were located by Burnham and Mattox and 13 nestlings banded (see Arctic 25(4):308-11 and 27(1):71-74 for background and preliminary reports of the first two years of the project). The project has banded 103 peregrine falcons (1972-78) of which 80 were color banded (red). All were banded as nestlings in the study area $\left(66^{\circ} 45^{\prime}-67^{\circ} 15^{\prime}\right.$ $\mathrm{N}, 50^{\circ}-52^{\circ} \mathrm{W}$ ) except three nestlings banded on Disko in 1974, one migrant HY male at Frederikshåb in 1975, and one AHY female banded in the study area in 1975. Of the 103 peregrines banded since 1972, four have been recovered (3.88\%). Two banded falcons were trapped and released by autumn banders at Cape Charles, Virginia (1974) and Cape May, New Jersey (1978). One peregrine banded in July 1974 was shot in Ecuador in December 1975. A fourth falcon, also banded in July 1974, was found dead in June 1975 in Egedesminde District, $137 \mathrm{~km}$ ( $85 \mathrm{mi}$.) northwest of its banding place.

In 1978 five team members travelled from USA to Søndre Strømfjord, Greenland, on July 18th to begin the survey. A sixth member (Heinrich) arrived on July 25th. Oar and Smylie surveyed the southeastern extremity of Angmalortup Nunâ, while Mattox, Belardo, and Riddle traversed parts of Ilivilik, west of Isortoqelven. A lone, adult female peregrine was observed for the fifth straight year at a cliff where young hatched successfully in 1972 and 1973. At four other cliffs which peregrines formerly occupied, the team found nothing. Peregrine falcons successfully produced young at three cliffs which had been occupied by gyrfalcons early in the survey years.

The 1978 team banded 17 nestling falcons at six nest cliffs. In addition, six young could not be banded because of age at two other cliffs. We therefore observed 23 young at eight producing eyries.

Of the 17 peregrines banded 10 were females, 7 males. Of 8 producing eyries, 3 had 4 young each, 2 had 3 young, 2 had 2 young, and one had one young. Age of nestlings varied considerably: on the same date one eyrie had 3 young 5-7 days old, whereas another eyrie had 3 young that were 35-40 days old. At neither eyrie could the young be banded.

\footnotetext{
'Ohio Department of Natural Resources.

${ }^{2}$ Comell Peregrine Fund West.

${ }^{3}$ Roscoe, Illinois.

${ }^{4}$ Chihuahuan Desert Research Institute.

sUniversity of Texas System Cancer Center.

'U.S. Fish and Wildlife Service.
} 
One recovery has been reported: a female nestling banded on July 24,1978 was trapped and released by band-banders at Cape May, New Jersey, on October 12, 1978 (Clark, pers. comm.).

The team used 56 minutes of helicopter time in a Bell-204 and $1 \mathrm{hr} .50 \mathrm{~min}$. in a Bell-206; in addition, a Piper Apache (1 hr. $25 \mathrm{~min}$.) and several boats were chartered to gain access to the survey area.

In all, 26 persons have now participated in this project, including several autumn banding attempts in southwest Greenland in 1975 and 1976. Main support since 1973 has been provided by the U.S. Army (Ecology Branch, Chemical Systems Lab., Aberdeen Proving Ground, MD, Dr. F. Prescott Ward, Chief). The Ministry of Greenland, Sukkertoppen Kommunalbestyrelsen, and Dr. Finn Salomonsen have kindly provided encouragement and assistance.

Table 1. Nestling Peregrine Falcons Hatched and Banded 1972 - 1978

\begin{tabular}{lcccccc} 
& $\begin{array}{c}\text { Hatched } \\
\text { Total } \\
\text { eyries }\end{array}$ & Young & $\begin{array}{c}\text { Young/ } \\
\text { eyrie }\end{array}$ & M & F & Total \\
\hline Year & 7 & 18 & 2.57 & 9 & 4 & 13 \\
1972 & 9 & 24 & 2.66 & 19 & 5 & 24 \\
1973 & 6 & 18 & 3.00 & 13 & 5 & 18 \\
$1974^{*}$ & 5 & 14 & 3.00 & 6 & 6 & 12 \\
1975 & 4 & 13 & 3.25 & 3 & 7 & 10 \\
1976 & 4 & 9 & 2.25 & 3 & 6 & 9 \\
1977 & 8 & 23 & 2.87 & 7 & 10 & 17 \\
1978 & & & & & & \\
\hline \hline & $43^{* *}$ & 117 & 2.72 & 59 & 42 & 101
\end{tabular}

*Incl. 3 nestlings banded on Disko outside survey area

** 12 different eyries (cliffs)

Table 2 - Total eyries by number of young hatched

\begin{tabular}{lccccc} 
Year & 4 young & 3 young & 2 young & 1 young & Total \\
\hline 1972 & 1 & 4 & - & 2 & 7 \\
1973 & 1 & 5 & 2 & 1 & 9 \\
1974 & 2 & $3^{*}$ & - & 1 & 6 \\
1975 & 1 & 2 & - & 2 & 5 \\
1976 & 1 & 3 & - & - & 4 \\
1977 & - & 2 & 1 & 1 & 4 \\
1978 & 3 & 2 & 2 & 1 & 8 \\
Total & 9 & 21 & 5 & 8 & 43 \\
Cliffs & $(20 \%)$ & $(49 \%)$ & $(11 \%)$ & $(18 \%)$ &
\end{tabular}

*Incl. one eyrie of 3 young on Disko 


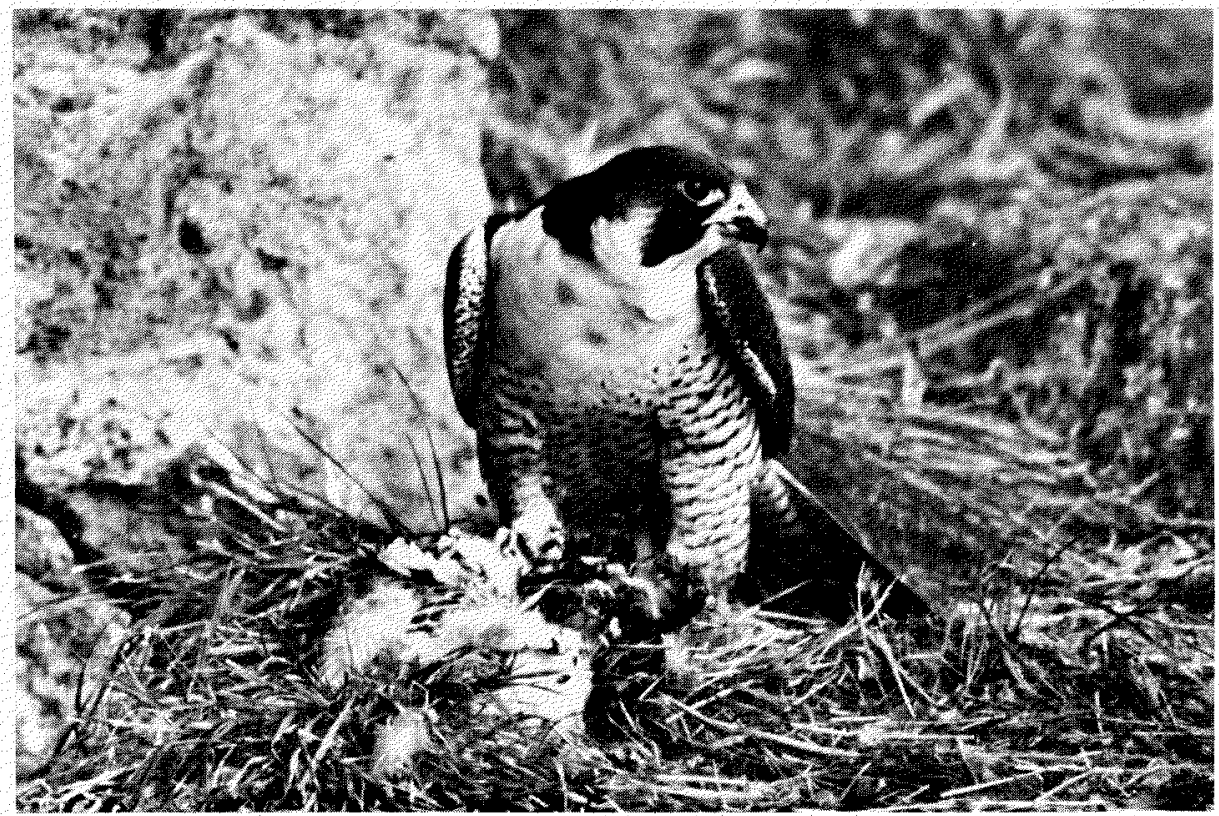

FIG. 1. Adult female peregrine falcon at an eyrie near Søndre Strømfjord, West Greenland, July 1972. Photo. D. M. Clement

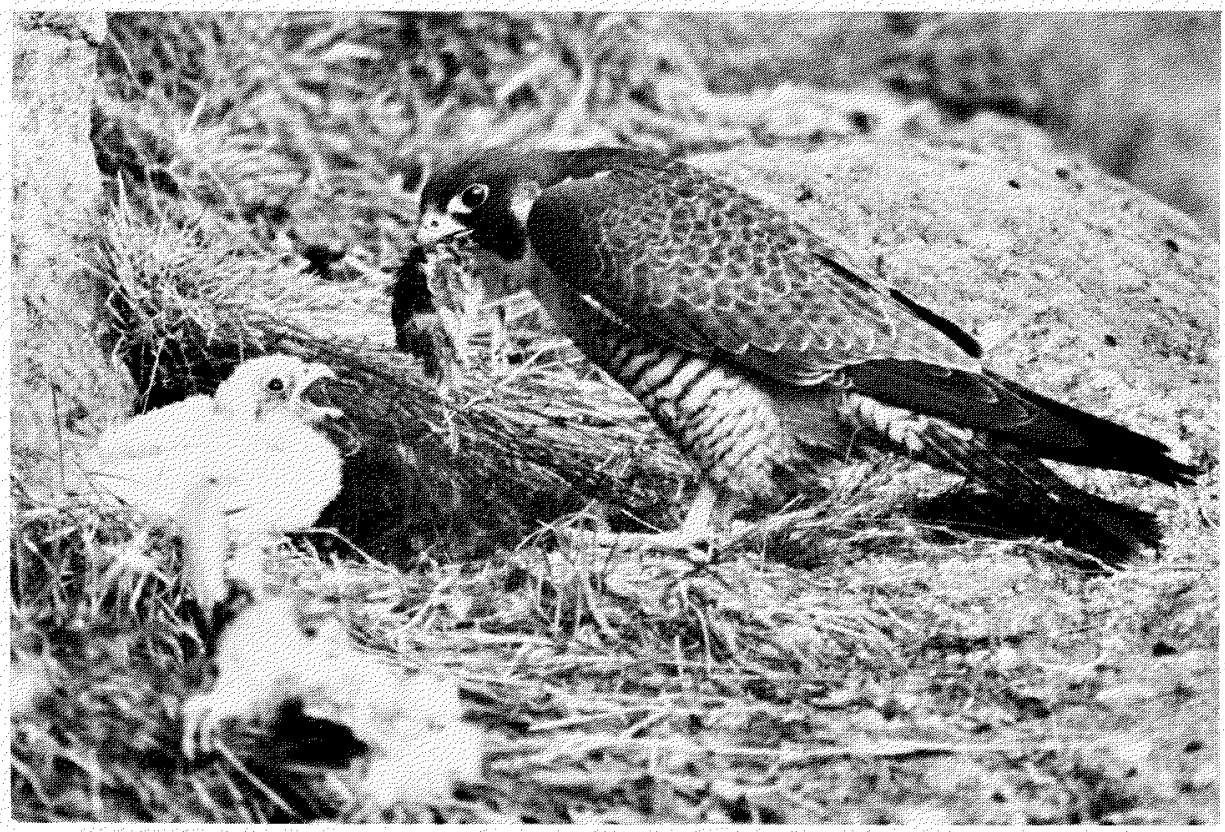

FIG. 2. Adult female peregrine falcon arrives at eyrie with prey to feed young. Near Sondre Strømfjord, West Greenland, July 1972. Photo: D. M. Clement 
Table 3 - Known Cliffs: Visited, Occupied, Producing, \% of visited cliffs producing, young/visited cliff.

\begin{tabular}{|c|c|c|c|c|c|c|c|}
\hline Year & Known* & Visited & Occupied ${ }^{* *}$ & Producing & $\begin{array}{c}\% \text { of } \\
\text { visited } \\
\text { cliffs } \\
\text { producing }\end{array}$ & $\begin{array}{l}\text { Young } \\
\text { Hatched }\end{array}$ & $\begin{array}{c}\text { Young/ } \\
\text { Visited } \\
\text { Cliff }\end{array}$ \\
\hline 1972 & 11 & 11 & 9 & 7 & 64 & 18 & 1.64 \\
\hline 1973 & 13 & 13 & 9 & 9 & 69 & 24 & 1.85 \\
\hline 1974 & 16 & 16 & 7 & 5 & 32 & 15 & 0.94 \\
\hline 1975 & 16 & 9 & 7 & 5 & 55 & 14 & 1.55 \\
\hline 1976 & 16 & 11 & 6 & 4 & 36 & 13 & 1.18 \\
\hline 1977 & 16 & 11 & 6 & 4 & 36 & 9 & 0.82 \\
\hline 1978 & $20^{* * *}$ & 17 & 10 & 8 & 47 & 23 & 1.35 \\
\hline
\end{tabular}

* Known to have been occupied by peregrine falcons (field observation, reliable local reports prior to 1972 , etc.).

** At least one adult present

***Includes 3 cliffs formerly occupied by gyrfalcons

\section{REFERENCES}

BURNHAM, W. A. 1975. Breeding biology and ecology of the peregrine falcon (Falco peregrinus) in West Greenland. M.S. thesis, Brigham Young Univ., unpubl. 57pp.

BURNHAM, W. A., JENKINS, M. A., WARD, F. P., MATTOX, W. G., CLEMENT, D. M., and HARRIS. J. T. 1974. Falcon research in Greenland, 1973. Arctic 27(1):71-74.

HARRIS, J. T. and CLEMENT, D. M. 1975. Greenland peregrines at their eyries: A behavioral study of the peregrine falcon. Meddelelser om Grønland 205(3):1-28.

JENKINS, M. A. 1978. Gyrfalcon nesting behavior from hatching to fledging. Auk 95(1): 122-127.

MATTOX, W. G. 1970a. Bird-banding in Greenland. Arctic 23(4):217-28.

1970b. Banding Gyrfalcons (Falco rusticolus, L.) in Greenland, 1967. Bird-banding 41(1):31-37.

. 1975. Bird of prey research in West Greenland, 1974. Polar Record 17(109):387-88.

, GRAHAM, R. A., BURNHAM, W. A., CLEMENT, D. M., and HARRIS, J. T. 1972. Peregrine falcon survey, West Greenland, 1972. Arctic 25(4):308-11.

WALKER, W. W., MATTOX, W. G., and RISEBROUGH, R. W. 1973. Pollutant and shell thickness determinations of peregrine eggs from West Greenland. Arctic 26(3):255-56. 